\title{
44101 - AN UNUSUAL CAUSE OF DARK BLOOD ASPIRATION DURING ARTERIAL CANNULATION: INTRAOPERATIVE DETECTION OF SULFHEMOGLOBINEMIA IN A PATIENT TAKING SUMATRIPTAN
}

Disclosure: Grants/research support - Dr Schwarz - 2006 CAS Research Award \& 2006 CAS/Abbott Laboratories Ltd Career Scientist Award in Anesthesia

\author{
Alana Flexman, University of British Columbia, Vancouver, BC, Canada; \\ Alana Flexman, University of British Columbia; \\ Stephan Schwarz, St Paul's Hospital, UBC; \\ Giuseppe Del Vicario, St Paul's Hospital, UBC;
}

PURPOSE: Sulfhemoglobinemia is a rare cause of intraoperative dyshemoglobinemia that may easily be confused with methemoglobinemia. Sulfhemoglobin is formed when a sulfur atom is incorporated irreversibly into the porphyrin ring of the heme group, which leads to oxidative denaturation and renders hemoglobin ineffective for oxygen transport $(1,2)$. It is caused by a variety of toxins and drugs and of these, sulfonamides, phenacetin, acetanilide and aniline derivatives have been implicated the most frequently $(1,2,3,4)$. The purpose of this case is to provide the first description of sulfhemoglobinemia associated with the sulfonamide-containing antimigraine drug, sumatriptan, presenting intraoperatively as unexplained dark discoloration of arterial blood.

CLINICAL FEATURES: The following case was compiled with the patient's consent. We describe a 42 year old male patient who presented for emergency bilateral lower extremity fasciotomies for compartment syndrome after falling asleep in the kneeling position for two hours. Intraoperatively, radial arterial catheterization yielded dark, discolored blood.

Intraoperative co-oximetry revealed arterial blood carboxyhemoglobin and methemoglobin concentrations of 1.17 and $2.99 \mathrm{~g} / \mathrm{L}$, respectively (fractions 0.023 and 0.009 ), and the analyzer displayed an alert to the presence of sulfhemoglobin. Confirmatory quantitative sulfhemoglobin analysis done postoperatively revealed a concentration of $2 \mathrm{~g} / \mathrm{L}$. Preoperatively, the patient had taken high doses of sumatriptan on a regular basis (200 mg per day), a sulfonamide-containing drug not previously associated with sulfhemoglobinemia. The patient stopped the sumatriptan postoperatively and recovered uneventfully. Repeat co-oximetry five weeks after discontinuation of sumatriptan detected no sulfhemoglobin. Extensive inquiry into the patient's past medical, medication and social history did not reveal any other likely causes of his sulfhemoglobinemia.

CONCLUSION: We report the first case of sulfhemoglobinemia associated with sumatriptan, presenting intraoperatively as dark arterial blood discoloration. Sulfhemoglobinemia should be considered in the differential diagnosis of unexplained perioperative dark blood discoloration, cyanosis and oxygen desaturation in the absence of hypoxemia, particularly in patients taking sulfonamide-containing drugs.

REFERENCES: 1. N Engl J Med 1984; 310: 1579-84. 2. N Engl J Med 1948; 239: 470-8. 3. Anesthesiology 2000; 93: 883-4. 4. West J Med 1998; 169: 386-9. 